\title{
Development of an Easy-to-Use Cryo-Electron Microscope for Simultaneous Observation of SEM and Transmission Images
}

\author{
Yoichi Ose ${ }^{1}$, Takeshi Sunaoshi ${ }^{1}$, Yusuke Tamba ${ }^{1}$, Yasuhira Nagakubo ${ }^{1}$, Junzo Azuma ${ }^{1}$, Ryuichiro \\ Tamochi $^{1}$, Akihiro Narita ${ }^{2}$, Tomoharu Matsumoto ${ }^{2}$, Eiji Usukura ${ }^{2}$, Jiro Usukura ${ }^{2}$ and Masako Osumi ${ }^{3}$ \\ 1. Hitachi High-Technologies Corporation, Hitachinaka-shi, Japan. \\ 2. Graduate School of Science, Nagoya University, Nagoya, Japan. \\ 3. Japan Women's University, Tokyo, Japan.
}

Researchers in all areas of medicine and biology have long awaited a user-friendly, low-acceleratingvoltage cryo-EM for a wide variety of applications. New low voltage cryo-scanning transmission electron microscope (STEM) has been developed based on conventional high-resolution SEM, which enables to observe a transmitted image and a secondary electron (SEM) image simultaneously in a fresh frozen state [1].

New cryo-transfer holder enables to observe specimens at $-190^{\circ} \mathrm{C}$ beneficial for low beam damage (Figure 1). We also added a vacuum system to convert the liquid nitrogen into a slush. In this way we succeeded ultimately in vibration-free observation (Figure 2). Specimen chamber is kept clean with anticontamination trap reached at $-210^{\circ} \mathrm{C}$ using slush nitrogen. Frozen specimens are mounted easily on the holder in liquid nitrogen by using a cryo-station. Simultaneous observation is advantageous to distinguish structures on surface or inside (Figure 3).

It is possible to observe biological specimens without any reduction of resolution because extremely high contrast is retained on focusing. High-resolution single particle analysis is easily obtained even in small number of specimens because correction of contrast transfer function is not necessary due to STEM optics [2]. Cryo-EM is not only useful for single-particle analysis, but is also an extremely powerful tool for conventional structural observations [3] [4]. In case of cell observation, cells cultured on carbon-coated grids are unroofed. Since some soluble components are eluted on unroofing, spatial architecture of cytoskeletal actin filaments and microtubules are observed at extremely high contrast.

\section{References:}

[1] E. Usukura et al, Sci. Rep. 6 (2016), p. 27472.

[2] T. Oda et al, Nature 457 (2009), p. 441.

[3] A. Al-Amoudi et al, EMBO J 23 (2004), p. 3583.

[4] J. Fontana \& A. Steven, Arch. Biochem. Biophy. 5891 (2015), p. 86. 


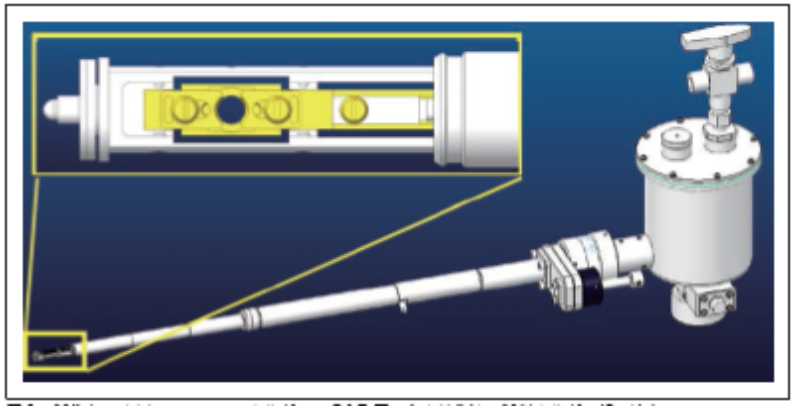

Figure 1. CAD diagram of the prototype cryo-transfer holder. The inset (upper left) shows an enlarged view of the specimen holder at the tip.
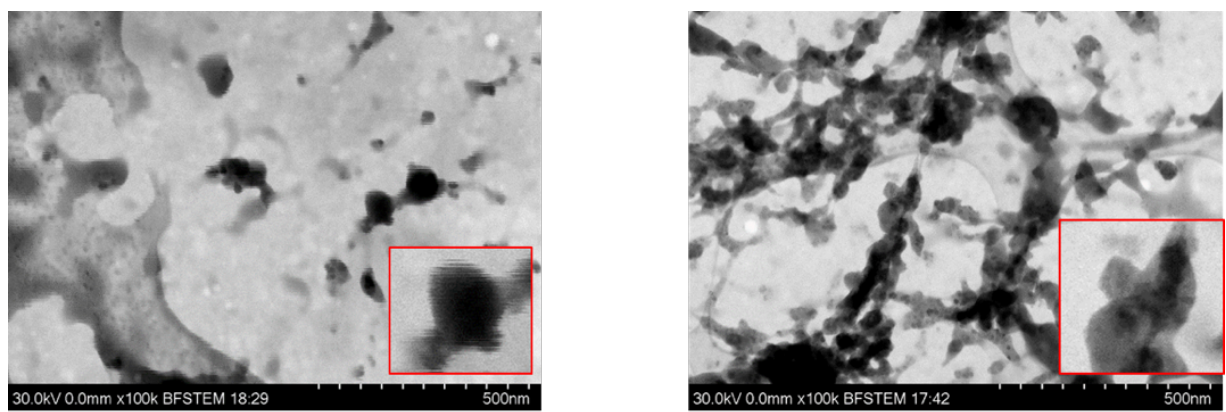

Figure 2. Images taken under liquid nitrogen cooling (left) and slush nitrogen cooling (right)
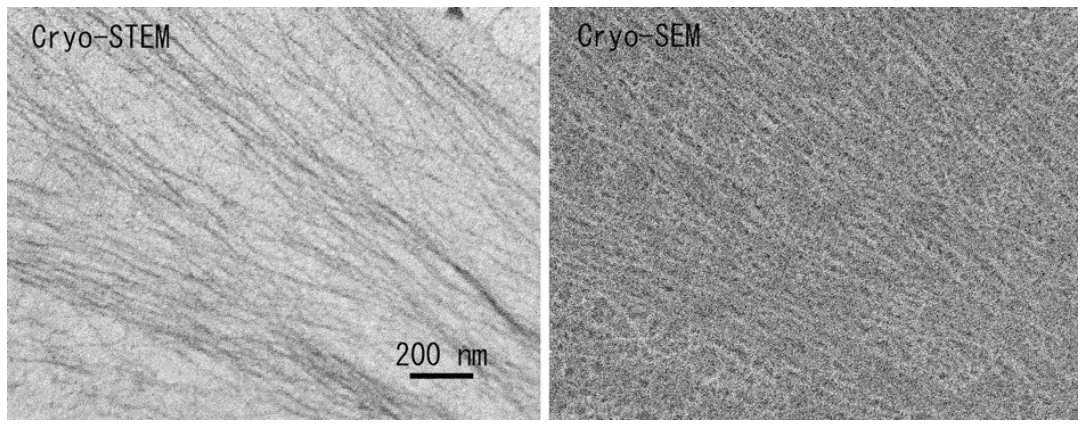

Figure 3. Simultaneously recorded cryo-STEM (left) and cryo-SEM (right) images

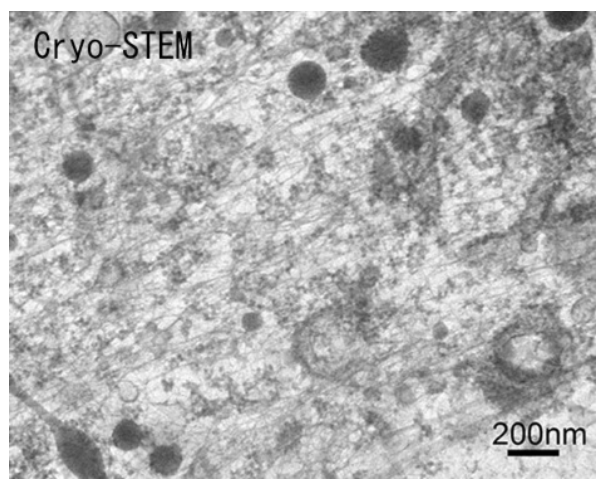

Figure 4. Cryo-STEM images of an unfixed sample after membrane removal 